ISSN 1392-3196 / e-ISSN 2335-8947

Zemdirbyste-Agriculture, vol. 104, No. 3 (2017), p. 259-266

DOI 10.13080/z-a.2017.104.033

\title{
Quantifying allelopathic effect of rapeseed on germination and seedling growth of maize under different salinity levels
}

\author{
Muhammad NAEEM ${ }^{1}$, Umair NISAR ${ }^{1}$, Farhan KHALID ${ }^{1}$, Azhar MEHMOOD ${ }^{1}$, \\ Hafiz Haider $\mathrm{ALI}^{2}$ \\ ${ }^{1}$ University College of Agriculture and Environmental Sciences, \\ The Islamia University of Bahawalpur \\ 63100 Punjab, Pakistan \\ E-mail: farhan.khalid@iub.edu.pk \\ ${ }^{2}$ University College of Agriculture, University of Sargodha \\ Sargodha, Punjab, Pakistan
}

\begin{abstract}
Brassica species have been reported as having allelopathic effect, reducing seed germination and emergence of subsequent cereal crops when grown in a rotation. Therefore, laboratory and greenhouse experiments were conducted to determine the allelopathic potential of rapeseed (Brassica napus L.) on germination and seedling growth of maize (Zea mays L.) under different salinity levels. Laboratory experiment consisted of two factors including different plant parts (root, shoot, leaf and whole plant) and their various concentrations $(0,5,10,15$ and $20 \%)$. In a greenhouse experiment three factors were studied including different salinity levels ( 0,4 and $8 \%$ ), crop residue concentrations $(0,2$ and $4 \%$ ) and decomposition time ( 2 and 4 weeks). The experiment was laid out in a completely randomized design with factorial arrangement where treatments were repeated five times for germination experiment conducted in Petri plates and three times for soil bioassay experiment conducted in pots. The results showed that extracts of different plant parts could not affect the germination, while different concentrations showed significant effect on maize seed germination. The interaction of extracts of different plant parts with different concentrations significantly affected the growth. Root and leaf extracts at 5\% concentration produced maximum root length, shoot length and plant dry weight as compared to other plant parts at other concentrations. For the second experiment or greenhouse experiment the results showed that neither plant parts nor decomposition period could influence germination significantly. However, salinity levels showed inhibitory effect on germination with increase in salt concentration. The rapeseed residue concentration of $2 \%$ with $4 \%$ salt concentration produced maximum plant fresh and dry weight. Rapeseed showed allelopathic potential on germination and seedling growth of maize, but this influence was decreased gradually with the increase of salinity.
\end{abstract}

Key words: allelopathy, Brassica napus, growth promotion, Zea mays.

\section{Introduction}

Brassica napus commonly known as rapeseed, belongs to the Brasscicaceae family, which produces fruiting bodies called pods. Brassica species of North America and Europe are commercially used as an oil seed and green manure crop but in western countries traditional rapeseed was considered unsuitable for food consumption either human or animals due to the presence of two naturally occurring toxicants in the seed, erucic acid and glucosinolates (Chauhan et al., 2007). Although B. napus is not native to Pakistan and its origin is reported either the Mediterranean areas or Northern Europe, even though it is profitably grown in Pakistan (Ali et al., 2012).

It has been reported that the plants in the vicinity are affected by several toxic compounds released from other weed residues (Nazir et al., 2007; Singh et al., 2007). Recently scientists have been giving more attention to allelopathic research due to weed-crop interaction (Singh et al., 2007), and numerous weeds have been identified for their allelopathic potential (Duke et al., 2007; Jabeen, Ahmed,2009).Allelopathic plants have potential to modify the growth of neighbouring plants by releasing secondary metabolites. Allelochemicals are toxic substances which restrain the growth of other plants (Batish et al., 2007; Asgharipour, Armin, 2010), therefore release of toxic chemicals from different plant parts ultimately have influence on plants in its vicinity. Plant extracts obtained from different crop residues influence crop growth and yield (Farooq et al., 2008). Brassica nigra seed releases

Please use the following format when citing the article:

Naeem M., Nisar U., Khalid F., Mehmood A., Ali H. H. 2017. Quantifying allelopathic effect of rapeseed on germination and seedling growth of maize under different salinity levels. Zemdirbyste-Agriculture, 104 (3): 259-266 DOI 10.13080/z-a.2017.104.033 
compounds which reduce wheat (Triticum aestivum L.) germination also releases allyl isothiocyanates which are supposed to be an important allelochemical (Al-Sherif et al., 2013). Mostly it is difficult to identify the nature of allelochemicals due to the lack of reliable techniques that can separate the allelopathic potential and plant interaction (He et al., 2012).

Germination and seedling characteristics are the most suitable and widely used criteria for examining salt tolerance of plants as they depict the ability of plants to germinate and most often become the limiting factor for crop production (Somani, 2007). Salinity affects the seed germination due to ionic stress and $\mathrm{NaCl}$ accumulation at toxic levels in plant tissues (Khodarahmpour et al., 2012). The inhibitory effect of increased salt concentration on germination rate and early seedling growth of four different vegetable plant species have also been reported by Jamil et al. (2006). Many studies report that seeds germinate well in distilled water and elevated salinity levels become limiting factor for seed germination and seedling development (Zehtab-Salmasi, 2008; Devkota, Jha, 2010). Salinity may affect the physiological and biochemical processes depending upon the developmental stage of the plant which ultimately results in lower biomass production. The tolerance of plants to such elevated salinity levels varies from species to species.

Mostly plant residues release phytotoxic compounds that show inhibitory effect on the growth of nearby plants. These phytotoxic compounds mostly contained phenolics that alter the soil structure and plant growth processes are significantly lowered due to altered soil quality. In addition to the phenolic compounds, salt concentrations also showed inhibitory effect on germination and seedling growth and this impact of salinity is highly dependent on type of genotype (Aliu et al., 2015). The reduction in maize seed germination, seedling growth and yield related parameters was also observed by Ouda et al. (2008).

Maize-oilseed rape rotation is a common rotation which is used in Pakistan; mostly growers incorporate the residues in soil after harvesting of rapeseed to improve the organic matter content of soil. It also influences the germination and growth of maize. The objective of the current study was to assess the effect of root, stem, leaf and whole plant allelopathic water extracts of rapeseed applied at different concentrations on maize germination and seedling growth. Further, the objective was also to assess the effect of decomposed rapeseed residue concentrations along with different salinity levels and decomposition period on maize germination and seedling growth.

\section{Materials and methods}

Collection of plants. The rapeseed (Brassica napus L.) plants were collected from different fields around the Bahawalpur, Southern Punjab, Pakistan $\left(29^{\circ} 23^{\prime} 44^{\prime \prime} \mathrm{N}, 71^{\circ} 41^{\prime} 1^{\prime \prime} \mathrm{E}\right)$ in October, 2015. The plants were dried at room temperature $\left(30 \pm 4^{\circ} \mathrm{C}\right)$ for seven days. The extracts obtained, as mentioned below, were applied in mid December 2015, when the experiments were conducted.

Preparation of water extracts. The plants were separated into leaf, stem, root and whole plant parts. Each plant part was divided into pieces. The dried pieces of the rapeseed plant (roots, stems, leaves and whole plant) were weighed and immersed in tap water at a ratio of 1:5 (w/v) at room temperature for $24 \mathrm{~h}$ for preparation of stock solution. The water extracts of the different parts of rapeseed were obtained by filtering through 10, 60 and 100-mesh sieves. After 24 hours, the solutions were filtered and the extracts were collected. These extracts were individually stored in clean bottles and tagged. Extract of leaf, root, shoot and whole plant were prepared in 1:5 (w/v) for the preparation of stock solution ( $20 \%$ concentration) and further diluted to prepare concentrations of 15,10 and $5 \%$ using parallel dilution technique $\left(\mathrm{C}_{1} \mathrm{~V}_{1}=\mathrm{C}_{2} \mathrm{~V}_{2}\right)$, whereas distilled water was used as control. Maize (hybrid 'Rhafan-2301') seeds were used to test the effect of rapeseed on their germination and early seedling growth.

Experiment No. 1: Laboratory bioassay. The experiment comprised two factors including extracts of different plant parts (root, stem, leaf and whole plant) and different concentrations $(0,5,10,15$ and $20 \%)$. This experiment was laid out in completely randomized design with factorial arrangements and five replications of the treatments. Ten seeds were placed on filter paper in $9 \mathrm{~cm}$ diameter Petri dishes. Before sowing, maize seeds were surface-sterilized with $1.5 \%(\mathrm{v} / \mathrm{v})$ sodium hypochlorite solution for $1 \mathrm{~min}$ and washed (three times; $3 \mathrm{~min} /$ wash) in distilled water. In each Petri dish, $10 \mathrm{~mL}$ of extract or distilled water were added as per treatment. To avoid the drying out of seeds throughout the incubation period, the Petri dishes were sealed with parafilm. During this period, the Petri dishes were observed daily and water or plant extracts were applied on daily basis.

Experiment No. 2: Greenhouse experiment. Experiment was carried out first in green house for the decomposition of the rapeseed residues and then in the Laboratory of Department of Agronomy, University College of Agriculture and Environmental Sciences, The Islamia University of Bahawalpur. Field soil was dried, crushed, mixed, and placed into $14 \mathrm{~cm}$ diameter plastic pots. This experiment was laid out in completely randomized design with factorial arrangement in pots and three replications of the treatments. The experiment was comprised of three factors including plant residues $(0,2$ and $4 \%)$, different $\mathrm{NaCl}$ concentrations $(0,4$ and $8 \%$ ) and two decomposition periods ( 2 and 4 weeks). The dried rapeseed plants were crushed and mixed with soil of these pots at the rate of 0,2 and $4 \%(\mathrm{w} / \mathrm{w})$ per pot and allowed to decompose for the prescribed period. To maintain electrical conductivity (EC) of soil 0, 4 and $8 \mathrm{~g}$ salt was added to pots. Then, 10 seeds of maize were sown in each pot. Water was supplied at regular basis. After 21 days, the seedlings were uprooted and washed with water. Seedling fresh and dry weight, length of roots and shoots were measured.

Observations recorded. For germination and greenhouse experiment data was collected on daily basis by recording the number of germinated or emerged seeds from treatments in each replication. Germination or emergence percentage was recorded by obtaining ratio of germinated or emerged and total seeds and converted in percent. The germination or emergence index was calculated as described by the AOSA Rules for Testing Seeds (1990). Mean germination or emergence time was calculated using the formula:

$$
\bar{t}=\frac{\sum_{i=1}^{k} n_{i} t_{i}}{\sum_{i=1}^{k} n_{i}}
$$

where $t_{i}$ is time from the start of the experiment to the $i^{\text {th }}$ observation, $n_{i}$ - number of seeds germinated or 
emerged in the $i^{\text {th }}$ time, and $k$ - last time of germination or emergence as cited by Ellis and Roberts (1980).

Time to $50 \%$ germination or emergence of seedlings was calculated according to the formula:

$$
T_{50}=\frac{t_{i}+\left\{\left(\frac{N}{2}\right)-n_{i}\right\}\left(t_{j}-t_{i}\right)}{\left(n_{j}-n_{i}\right)},
$$

where $N$ is the final number of germination or emergence, $n_{i}$ and $n_{j}$ are cumulative number of seeds germinated or emerged by adjacent counts at times $t_{i}$ and $t_{j}$, when $n_{i}<\frac{N}{2}<n_{j}$ (Coolbear et al., 1984).

Synchrony of germination or emergence was calculated by the expression $Z=\frac{\sum_{i=1}^{k} c_{n i, 2}}{c_{\sum_{i-1}^{k} n i, 2}}$ being $C_{n i, 2}=\frac{n_{i}\left(n_{i}-1\right)}{2}$,

where $\mathrm{C}_{n i, 2}$ is combination of the seeds germinated or emerged in the $i^{\text {th }}$ time, two by two, $n_{i}-$ number of seeds germinated or emerged in the $i^{\text {th }}$ time.

Uncertainty of the germination or emergence process was calculated by the following formula:

$$
U=-\sum_{i=1}^{k} f_{i} \log _{2} f_{i} \text { being } f_{i}=\frac{n_{i}}{\sum_{i=1}^{k} n_{i}},
$$

where $n_{i}$ is number of seeds germinated or emerged in the $i^{\text {th }}$ time, $k$ - last day of observation (Ranal et al., 2009). In the soil bioassay experiment, fresh and dry weights in grams were obtained from each replication for root and shoot, whereas lengths were measured in $\mathrm{cm}$ using a ruler.

Statistical analysis. The data were analyzed using Fisher's analysis of variance technique and treatments' means were compared applying least significant difference test (LSD) at 5\% probability level (Steel et al., 1997).

\section{Results}

Extracts of plant parts and different concentrations. The concentrations of different plant extracts showed significant effect on germination while plant parts did not significantly affect it (Table 1). It has been recorded that with increase in concentration germination percentage subsequently reduced. The highest germination percentage, time to $50 \%$ germination and germination index was in control treatment. Although germination was inhibited under different concentrations, maximum mean germination time (MGT) was recorded in the control treatment giving lowest mean germination time where $10 \%$ extract concentration was used. The highest synchronization index was recorded in 5\% extract concentration and was also similar to the control treatment showing more uniformity of germination with decrease in concentration contrary to uncertainty of germination process that increased with increase in concentration.

Table 1. Effect of aqueous extracts of different rapeseed plant parts and their different concentrations on the germination

\begin{tabular}{|c|c|c|c|c|c|c|}
\hline Treatments & $\begin{array}{c}\text { Germination } \\
\%\end{array}$ & $\begin{array}{c}\text { Mean } \\
\text { germination } \\
\text { time, days }\end{array}$ & $\begin{array}{l}\text { Germination } \\
\text { index }\end{array}$ & $\begin{array}{l}\text { Time to } 50 \% \\
\text { germination, } \\
\text { days }\end{array}$ & $\begin{array}{c}\text { Synchronization } \\
\text { index }\end{array}$ & $\begin{array}{c}\text { Uncertainty of } \\
\text { germination process, } \\
\text { bit }\end{array}$ \\
\hline \multicolumn{7}{|c|}{ Plant extracts $(E)$} \\
\hline Root & 81.33 & 4.85 & 1.79 & 4.32 & 0.15 & 2.02 \\
\hline Stem & 81.33 & 4.65 & 1.86 & 4.07 & 0.17 & 1.90 \\
\hline Leaf & 80.00 & 4.66 & 1.82 & 4.08 & 0.16 & 1.95 \\
\hline Whole plant & 81.33 & 4.64 & 1.86 & 4.07 & 0.17 & 1.92 \\
\hline LSD & ns & ns & ns & ns & ns & ns \\
\hline \multicolumn{7}{|c|}{ Concentrations (C) } \\
\hline $0 \%$ (control) & $100.00 \mathrm{~A}$ & $4.96 \mathrm{~A}$ & $2.11 \mathrm{~A}$ & $4.39 \mathrm{~A}$ & $0.19 \mathrm{AB}$ & $1.93 \mathrm{AB}$ \\
\hline $5 \%$ & $88.33 \mathrm{~B}$ & $4.74 \mathrm{~B}$ & $1.95 \mathrm{~B}$ & 4.19 AB & $0.21 \mathrm{~A}$ & $1.85 \mathrm{~B}$ \\
\hline $10 \%$ & $82.50 \mathrm{C}$ & $4.51 \mathrm{C}$ & $1.99 \mathrm{AB}$ & $3.80 \mathrm{C}$ & $0.15 \mathrm{C}$ & $2.05 \mathrm{~A}$ \\
\hline $15 \%$ & $70.00 \mathrm{D}$ & $4.67 \mathrm{BC}$ & $1.62 \mathrm{C}$ & $4.31 \mathrm{~A}$ & $0.16 \mathrm{BC}$ & $1.86 \mathrm{~B}$ \\
\hline $20 \%$ & $64.17 \mathrm{E}$ & $4.63 \mathrm{BC}$ & $1.50 \mathrm{C}$ & $4.00 \mathrm{BC}$ & $0.11 \mathrm{D}$ & $2.06 \mathrm{~A}$ \\
\hline LSD & 5.312 & 0.202 & 0.144 & 0.205 & 0.033 & 1.778 \\
\hline
\end{tabular}
of maize

Note. Mean values with a different letter are significantly different at $5 \%$ level; ns - not significant at 5\% level.

Both extracts from different plant parts and different concentrations of these extracts significantly affected the root length, shoot length, plant fresh weight and plant dry weight (Table 2). Maximum root length, shoot length, plant fresh and dry weight were recorded when root extract was used.

This was also similar for whole plant fresh weight where whole plant extract was used. The interaction effect of different concentrations and plant parts showed that using root extracts of $10 \%$ concentration produced maximum root and shoot length and this was also similar to plant fresh and dry weight. However, maximum plant fresh and dry weight was obtained when no extract was applied and 5\% leaf extract was used, respectively (Table 2).

Brassica residue concentration, salinity level and decomposition period. The results obtained from experiment No. 2 showed that plant residues and decomposition period did not affect the emergence when maize seeds were sown in pots. However, salinity levels significantly affected these attributes by giving maximum emergence percentage, emergence index and synchronization index in control at $0 \%$ salinity level (Table 3). This is also indicated by mean emergence time, time to $50 \%$ emergence and uncertainty of emergence process which was lower in control where no application of residues was carried out and subsequently increased with increase in salinity level. However, the interaction among plant residues, salinity levels and decomposition period did not affect emergence attributes significantly.

Brassica residues concentrations significantly improved the shoot length, plant fresh weight. Maximum shoot length, plant fresh and dry weight were obtained when 4\% Brassica residue concentration was used and 
Table 2. Effect of aqueous extracts of different plant parts and their different concentrations on the growth of maize

\begin{tabular}{|c|c|c|c|c|c|}
\hline \multicolumn{2}{|c|}{ Treatments } & Root length cm & Shoot length $\mathrm{cm}$ & Plant fresh weight $g$ & Plant dry weight g \\
\hline \multicolumn{6}{|c|}{ Plant extracts $(\mathrm{E})$} \\
\hline \multicolumn{2}{|c|}{ Root } & $9.02 \mathrm{~A}$ & $5.88 \mathrm{~A}$ & $0.85 \mathrm{~A}$ & $0.68 \mathrm{~A}$ \\
\hline \multicolumn{2}{|c|}{ Stem } & $8.91 \mathrm{C}$ & $5.79 \mathrm{C}$ & $0.74 \mathrm{C}$ & $0.59 \mathrm{C}$ \\
\hline \multicolumn{2}{|c|}{ Leaf } & $8.97 \mathrm{~B}$ & $5.85 \mathrm{~B}$ & $0.81 \mathrm{~B}$ & $0.65 \mathrm{~B}$ \\
\hline \multicolumn{2}{|c|}{ Whole plant } & $8.98 \mathrm{~B}$ & $5.86 \mathrm{~B}$ & $0.84 \mathrm{~A}$ & $0.66 \mathrm{~B}$ \\
\hline \multicolumn{2}{|c|}{ LSD } & 0.044 & 0.014 & 0.017 & 0.014 \\
\hline \multicolumn{6}{|c|}{ Concentrations $(\mathrm{C})$} \\
\hline \multicolumn{2}{|c|}{$0 \%$ (control) } & $9.02 \mathrm{~A}$ & $5.90 \mathrm{~A}$ & $0.93 \mathrm{~A}$ & $0.70 \mathrm{~A}$ \\
\hline \multicolumn{2}{|c|}{$5 \%$} & $8.98 \mathrm{~B}$ & $5.86 \mathrm{~B}$ & $0.84 \mathrm{~B}$ & $0.66 \mathrm{~B}$ \\
\hline \multicolumn{2}{|c|}{$10 \%$} & $8.98 \mathrm{~B}$ & $5.86 \mathrm{~B}$ & $0.81 \mathrm{C}$ & $0.66 \mathrm{~B}$ \\
\hline \multicolumn{2}{|c|}{$15 \%$} & $8.96 \mathrm{~B}$ & $5.84 \mathrm{~B}$ & $0.78 \mathrm{D}$ & $0.64 \mathrm{C}$ \\
\hline \multicolumn{2}{|c|}{$20 \%$} & $8.88 \mathrm{C}$ & $5.76 \mathrm{C}$ & $0.69 \mathrm{E}$ & $0.56 \mathrm{D}$ \\
\hline \multicolumn{2}{|c|}{ LSD } & 0.016 & 0.016 & 0.019 & 0.015 \\
\hline \multicolumn{6}{|c|}{$\mathrm{E} \times \mathrm{C}$ interaction } \\
\hline \multirow{5}{*}{ Root extract } & $0 \%$ (control) & $9.02 \mathrm{AB}$ & $5.90 \mathrm{AB}$ & $0.93 \mathrm{~A}$ & $0.70 \mathrm{BC}$ \\
\hline & $5 \%$ & $9.03 \mathrm{AB}$ & $5.91 \mathrm{AB}$ & $0.90 \mathrm{AB}$ & $0.71 \mathrm{ABC}$ \\
\hline & $10 \%$ & $9.05 \mathrm{~A}$ & $5.93 \mathrm{~A}$ & $0.90 \mathrm{AB}$ & $0.73 \mathrm{AB}$ \\
\hline & $15 \%$ & $9.00 \mathrm{BC}$ & $5.88 \mathrm{BC}$ & $0.83 \mathrm{DE}$ & $0.68 \mathrm{CD}$ \\
\hline & $20 \%$ & $8.90 \mathrm{FG}$ & $5.78 \mathrm{FG}$ & $0.72 \mathrm{HI}$ & $0.59 \mathrm{G}$ \\
\hline \multirow{5}{*}{ Stem extract } & $0 \%$ (control) & $9.02 \mathrm{AB}$ & $5.90 \mathrm{AB}$ & $0.93 \mathrm{~A}$ & $0.70 \mathrm{BC}$ \\
\hline & $5 \%$ & $8.87 \mathrm{GH}$ & $5.75 \mathrm{GH}$ & $0.70 \mathrm{I}$ & $0.55 \mathrm{H}$ \\
\hline & $10 \%$ & 8.94 DE & $5.82 \mathrm{DE}$ & $0.77 \mathrm{FG}$ & $0.62 \mathrm{EF}$ \\
\hline & $15 \%$ & $8.87 \mathrm{GH}$ & $5.75 \mathrm{GH}$ & $0.65 \mathrm{~J}$ & $0.55 \mathrm{H}$ \\
\hline & $20 \%$ & $8.86 \mathrm{H}$ & $5.74 \mathrm{H}$ & $0.63 \mathrm{~J}$ & $0.54 \mathrm{H}$ \\
\hline \multirow{5}{*}{ Leaf extract } & $0 \%$ (control) & $9.02 \mathrm{AB}$ & $5.90 \mathrm{AB}$ & $0.93 \mathrm{~A}$ & $0.70 \mathrm{BC}$ \\
\hline & $5 \%$ & $9.05 \mathrm{~A}$ & $5.93 \mathrm{~A}$ & $0.88 \mathrm{BC}$ & $0.73 \mathrm{~A}$ \\
\hline & $10 \%$ & $8.92 \mathrm{EF}$ & $5.80 \mathrm{EF}$ & $0.74 \mathrm{GH}$ & $0.60 \mathrm{FG}$ \\
\hline & $15 \%$ & $9.02 \mathrm{AB}$ & $5.90 \mathrm{AB}$ & $0.85 \mathrm{CD}$ & $0.70 \mathrm{BC}$ \\
\hline & $20 \%$ & $8.86 \mathrm{H}$ & $5.74 \mathrm{H}$ & $0.66 \mathrm{~J}$ & $0.54 \mathrm{H}$ \\
\hline \multirow{6}{*}{$\begin{array}{c}\text { Whole plant } \\
\text { extract }\end{array}$} & $0 \%$ (control) & $9.02 \mathrm{AB}$ & $5.90 \mathrm{AB}$ & $0.93 \mathrm{~A}$ & $0.70 \mathrm{BC}$ \\
\hline & $5 \%$ & $8.97 \mathrm{C}$ & $5.85 \mathrm{C}$ & $0.89 \mathrm{AB}$ & $0.65 \mathrm{DE}$ \\
\hline & $10 \%$ & $9.01 \mathrm{~B}$ & $5.89 \mathrm{~B}$ & $0.85 \mathrm{CD}$ & $0.70 \mathrm{BC}$ \\
\hline & $15 \%$ & $8.97 \mathrm{CD}$ & $5.85 \mathrm{CD}$ & $0.80 \mathrm{EF}$ & $0.65 \mathrm{DE}$ \\
\hline & $20 \%$ & $8.91 \mathrm{EF}$ & $5.79 \mathrm{EF}$ & $0.73 \mathrm{HI}$ & $0.59 \mathrm{G}$ \\
\hline & LSD & 0.032 & 0.032 & 0.038 & 0.031 \\
\hline
\end{tabular}

Note. Mean values with a different letter are significantly different at $5 \%$ level.

Table 3. Effect of aqueous extracts of different residue concentrations, salinity levels and decomposition period on the emergence of maize

\begin{tabular}{|c|c|c|c|c|c|c|}
\hline Treatments & $\begin{array}{c}\text { Emergence } \\
\%\end{array}$ & $\begin{array}{c}\text { Mean } \\
\text { emergence time, } \\
\text { days }\end{array}$ & $\begin{array}{l}\text { Emergence } \\
\text { index }\end{array}$ & $\begin{array}{c}\text { Time to } 50 \% \\
\text { emergence, } \\
\text { days }\end{array}$ & $\begin{array}{l}\text { Synchronization } \\
\text { index }\end{array}$ & $\begin{array}{c}\text { Uncertainty of } \\
\text { emergence process, } \\
\text { bit }\end{array}$ \\
\hline \multicolumn{7}{|c|}{ Residues concentration } \\
\hline $0 \%$ (control) & 90.55 & 7.37 & 1.32 & 6.37 & 0.13 & 2.29 \\
\hline $2 \%$ & 88.33 & 7.35 & 1.28 & 6.48 & 0.17 & 2.10 \\
\hline $4 \%$ & 91.66 & 7.48 & 1.26 & 6.52 & 0.17 & 2.04 \\
\hline LSD & ns & $\mathrm{ns}$ & ns & ns & $\mathrm{ns}$ & ns \\
\hline \multicolumn{7}{|c|}{ Salinity levels } \\
\hline $0 \%$ (control) & $98.33 \mathrm{~A}$ & $7.13 \mathrm{~B}$ & $1.55 \mathrm{~A}$ & $6.16 \mathrm{~B}$ & $0.18 \mathrm{~A}$ & $2.17 \mathrm{AB}$ \\
\hline $4 \%$ & $94.44 \mathrm{~A}$ & $7.22 \mathrm{~B}$ & $1.41 \mathrm{~B}$ & $6.14 \mathrm{~B}$ & $0.19 \mathrm{~A}$ & $1.95 \mathrm{~B}$ \\
\hline $8 \%$ & $77.77 \mathrm{~B}$ & $7.84 \mathrm{~A}$ & $0.89 \mathrm{C}$ & $7.07 \mathrm{~A}$ & $0.10 \mathrm{~B}$ & $2.31 \mathrm{~A}$ \\
\hline LSD & 4.073 & 0.314 & 0.110 & 0.420 & 0.040 & 2.31 \\
\hline \multicolumn{7}{|c|}{ Decomposition period } \\
\hline 2 weeks & 90.00 & 7.40 & 1.28 & 6.50 & 0.15 & 2.18 \\
\hline 4 weeks & 90.33 & 7.39 & 1.29 & 6.40 & 0.17 & 2.11 \\
\hline LSD & ns & ns & ns & $\mathrm{ns}$ & ns & ns \\
\hline
\end{tabular}

Note. Mean values with a different letter are significantly different at $5 \%$ level; ns - not significant at $5 \%$ level.

reduced subsequently at lower residues concentration (Table 4). Salinity levels significantly affected the root and shoot length while plant fresh and dry weight were not affected at different salt concentrations. Root and shoot length decreased with increase in salt concentration and maximum root and shoot length was recorded in control where no salt was applied. Maximum root length, plant fresh weight and plant dry weight was recorded at
2 weeks decomposition period when compared with 4 weeks decomposition period. Increase in decomposition period reduced the growth attributes of maize plants.

The interaction among Brassica residue concentrations, salinity levels and decomposition period showed significant effect on growth of maize plants except shoot length (Table 5). 
Table 4. Effect of aqueous extracts of different residue concentrations, salinity levels and decomposition period on the growth attributes of maize

\begin{tabular}{|c|c|c|c|c|}
\hline Treatments & $\begin{array}{l}\text { Root length } \\
\mathrm{cm}\end{array}$ & $\begin{array}{c}\text { Shoot length } \\
\mathrm{cm}\end{array}$ & $\begin{array}{l}\text { Plant fresh weight } \\
\mathrm{g}\end{array}$ & $\begin{array}{c}\text { Plant dry weight } \\
\text { g }\end{array}$ \\
\hline \multicolumn{5}{|c|}{ Residues concentration } \\
\hline $0 \%$ (control) & 19.50 & $11.02 \mathrm{~B}$ & $1.67 \mathrm{~B}$ & $1.32 \mathrm{~B}$ \\
\hline $2 \%$ & 19.36 & $12.47 \mathrm{AB}$ & $1.68 \mathrm{~B}$ & $1.33 \mathrm{~B}$ \\
\hline $4 \%$ & 20.13 & $14.37 \mathrm{~A}$ & $2.08 \mathrm{~A}$ & $1.73 \mathrm{~A}$ \\
\hline LSD & ns & 2.282 & 0.355 & 0.355 \\
\hline \multicolumn{5}{|c|}{ Salinity levels } \\
\hline $0 \%$ (control) & $22.10 \mathrm{~A}$ & $13.79 \mathrm{~A}$ & 1.65 & 1.30 \\
\hline $4 \%$ & $19.83 \mathrm{AB}$ & $13.60 \mathrm{~A}$ & 1.92 & 1.57 \\
\hline $8 \%$ & $17.05 \mathrm{~B}$ & $10.47 \mathrm{~B}$ & 1.85 & 1.50 \\
\hline LSD & 3.254 & 2.282 & ns & $\mathrm{ns}$ \\
\hline \multicolumn{5}{|c|}{ Decomposition period } \\
\hline 2 weeks & $22.70 \mathrm{~A}$ & 12.75 & $2.35 \mathrm{~A}$ & $2.00 \mathrm{~A}$ \\
\hline 4 weeks & $19.36 \mathrm{~B}$ & 12.49 & $1.27 \mathrm{~B}$ & $0.92 \mathrm{~B}$ \\
\hline LSD & 2.657 & ns & 0.290 & 0.290 \\
\hline
\end{tabular}

Note. Mean values with a different letter are significantly different at $5 \%$ level; ns - not significant at $5 \%$ level.

Table 5. Effect of aqueous extracts of different decomposition period (DP), residue concentrations (RC) and salinity levels on the growth of maize

\begin{tabular}{|c|c|c|c|c|c|c|}
\hline \multicolumn{3}{|c|}{ Treatments } & $\begin{array}{l}\text { Root length } \\
\mathrm{cm}\end{array}$ & $\begin{array}{c}\text { Shoot length } \\
\mathrm{cm}\end{array}$ & $\begin{array}{c}\text { Plant fresh weight } \\
\mathrm{g}\end{array}$ & $\begin{array}{l}\text { Plant dry weight } \\
\text { g }\end{array}$ \\
\hline \multirow{9}{*}{$\begin{array}{c}2 \text { weeks } \\
\text { DP }\end{array}$} & \multirow{3}{*}{$0 \% \mathrm{RC}$} & $0 \%$ salinity level & $26.16 \mathrm{AB}$ & 31.23 & $2.23 \mathrm{BCDE}$ & $1.88 \mathrm{BCDE}$ \\
\hline & & $4 \%$ salinity level & $22.26 \mathrm{ABCD}$ & 25.42 & 2.06 BCDEF & 1.71 BCDEF \\
\hline & & $8 \%$ salinity level & $13.93 \mathrm{EFG}$ & 13.51 & $2.16 \mathrm{BCDE}$ & $1.81 \mathrm{BCDE}$ \\
\hline & \multirow{3}{*}{$2 \% \mathrm{RC}$} & $0 \%$ salinity level & 19.36 BCDEF & 24.63 & $1.20 \mathrm{FG}$ & $0.85 \mathrm{FG}$ \\
\hline & & $4 \%$ salinity level & $26.50 \mathrm{AB}$ & 36.59 & $3.26 \mathrm{~A}$ & $2.91 \mathrm{~A}$ \\
\hline & & $8 \%$ salinity level & 16.36 DEFG & 18.05 & $2.33 \mathrm{BCD}$ & $1.98 \mathrm{BCD}$ \\
\hline & \multirow{3}{*}{$4 \% \mathrm{RC}$} & $0 \%$ salinity level & $28.56 \mathrm{~A}$ & 36.33 & $2.66 \mathrm{AB}$ & $2.31 \mathrm{AB}$ \\
\hline & & $4 \%$ salinity level & $26.03 \mathrm{AB}$ & 35.60 & $2.53 \mathrm{ABC}$ & $2.18 \mathrm{ABC}$ \\
\hline & & $8 \%$ salinity level & $25.16 \mathrm{ABC}$ & 26.46 & $2.70 \mathrm{AB}$ & $2.35 \mathrm{AB}$ \\
\hline \multirow{9}{*}{$\begin{array}{c}4 \text { weeks } \\
\text { DP }\end{array}$} & \multirow{3}{*}{$0 \% \mathrm{RC}$} & $0 \%$ salinity level & 19.26 BCDEF & 22.43 & $0.96 \mathrm{G}$ & $0.61 \mathrm{G}$ \\
\hline & & $4 \%$ salinity level & $20.96 \mathrm{ABCDE}$ & 25.80 & 1.46 DEFG & 1.11 DEFG \\
\hline & & $8 \%$ salinity level & 14.40 DEFG & 18.17 & $1.13 \mathrm{G}$ & $0.78 \mathrm{G}$ \\
\hline & \multirow{3}{*}{$2 \% \mathrm{RC}$} & $0 \%$ salinity level & $24.73 \mathrm{ABC}$ & 33.20 & $1.36 \mathrm{EFG}$ & $1.01 \mathrm{EFG}$ \\
\hline & & $4 \%$ salinity level & $11.26 \mathrm{G}$ & 14.90 & $0.86 \mathrm{G}$ & $0.51 \mathrm{G}$ \\
\hline & & $8 \%$ salinity level & $17.93 \mathrm{CDEFG}$ & 15.96 & $1.06 \mathrm{G}$ & $0.71 \mathrm{G}$ \\
\hline & \multirow{3}{*}{$4 \% \mathrm{RC}$} & $0 \%$ salinity level & $14.50 \mathrm{DEFG}$ & 24.43 & $1.50 \mathrm{DEFG}$ & $1.15 \mathrm{DEFG}$ \\
\hline & & $4 \%$ salinity level & $12.00 \mathrm{FG}$ & 18.76 & $1.36 \mathrm{EFG}$ & $1.01 \mathrm{EFG}$ \\
\hline & & $8 \%$ salinity level & 14.53 DEFG & 17.09 & $1.73 \mathrm{CDEFG}$ & 1.38 CDEFG \\
\hline \multicolumn{3}{|c|}{ LSD } & 7.972 & ns & 0.870 & 0.870 \\
\hline
\end{tabular}

Note. Mean values with a different letter are significantly different at $5 \%$ level; ns - not significant at $5 \%$ level.

Maximum root length was recorded when 4\% Brassica residue concentration after 2 weeks decomposition period at $0 \%$ salinity level was used and this was also similar for plant fresh and dry weights. However, maximum plant fresh and dry weight was recorded at $2 \%$ Brassica residues concentration after 2 weeks of decomposition period when $4 \%$ salt concentration was used.

\section{Discussion}

Application of different concentrations of rapeseed residue extracts reduced germination percentage, mean germination time, germination index and time to $50 \%$ germination with increase in concentration.
Inhibitory effect on seedling growth of maize was recorded with increase in extracts concentration. Seed germination and early seedling growth stages are more sensitive to stress conditions for many crops (Rahimi, 2013). So, germinability, mean germination time and synchronization index are essential attributes that indicate healthier and successful crop production.

The results obtained in the current study indicated that extracts of Brassica significantly decreased the germination of maize. Similar results were reported by Haddadchi and Gerivani (2009), who explained that phenolic extracts of rapeseed (Brassica napus L.) contain chemical substances that decreased the germination and seedling growth of soybean (Glycin $\max \mathrm{L}$.). The increase in $\mathrm{NaCl}$ concentration inhibited the 
germination attributes of maize crop. It is well supported by the findings of Chachar et al. (2008) who reported that salt stress inhibits seed germination by limiting water absorption.

The extracts of different plant parts and their different concentrations significantly affected the growth attributes of maize plants. Plant parts and concentrations showed inhibitory effect of growthattributes whichreduces root and shoot length and plant fresh and dry weight with increase in concentration. Results are supported by Ullah et al. (2014) who explained that the presence of phenolic compounds showed inhibitory effects on seed germination percentage and activity of enzyme lipase of germinating seeds of canola. Presence of glucosinolates in Brassica species make them strongly allelopathic crops (Jafariehyazdi, Javidfar, 2011). However, contradictory effects of Brassica extracts were observed by Walsh et al. (2014) who reported that germination rate of wild oat (Avena fatua L.), flax (Linum usitatissimum L.) and radish (Raphanus sativus L.) was not significantly decreased by extract concentrations. Similar results were also reported by Iqbal et al. (2014) that foliar application of low concentrations of aqueous extracts such as moringa (Moringa oleifera L.) and Brassica can be used as growth promoter. Rigon et al. (2012) also reported that germinating percentage of Phaseolus vulgaris was not influenced by increasing the concentrations of rapeseed. Compound glucosinolates present in Brassica species suppress the seed germination of many plants, inhibitory results of glucosinolate released from Brassica species were also observed by Vaughn et al. (2006), who concluded that presence of water soluble compounds decreased the seed germination at all concentrations of Indian mustard (Brassica juncea L.), money plant (Epipremnum aureum L.) and field pennycress (Thlaspi arvense L.) seed meals.

Effect of crop residues on maize growth traits showed non-significant results. Growth parameters are directly related to germination. Growth traits lead to a successful crop production. However, crop residues increase the growth parameters in some instance, results supported by Messiha et al. (2013) illustrated that growth characteristics of maize increased at 25 and $50 \mathrm{~g} \mathrm{~kg}^{-1}$ soil residual concentration as compared to controls. Crop residues of rapeseed have strong allelopathic effect on germination and growth of other plant. It was found that decomposed residues of rapeseed release secondary metabolites which significantly suppress the growth of other plants (Zaji, Majd, 2011).

Additional factor of salt stress significantly showed drastic results on growth attributes of maize. Seed emergence and early seedling growth stages are more sensitive to salt stress. Our results indicated that factor of salinity with crop residues significantly reduced the germination of maize. A clear reduction in shoot length, root length and whole plant length were observed as compared to control treatment. The seedling growth was totally suppressed in salinity stress and crop residue combinations. Similar results were observed by Alam and Shaikh (2007) in wheat crop.

\section{Conclusion}

Rapeseed (Brassica napus L.) extracts showed allelopathic potential on germination as well as growth of maize. However, using different plant parts of rapeseed showed no differences for maize seed germination characteristics. Root extract improved the seedling growth attributes by enhancing root and shoot length and plant fresh and dry weight compared to stem, leaf and whole plant extracts. With increase in extract concentration maize seed germination was consistently reduced. Allelopathic water extracts obtained from rapeseed roots at $5 \%$ concentration consistently performed better in improving the seedling growth of maize than stem, leaf and whole plant water extracts at other concentrations.

In pot experiment, rapeseed residue concentrations and decomposition period alone did not affect the emergence of maize plants. However, salinity induced by $\mathrm{NaCl}$ concentration of $4 \%$ showed enhanced emergence and seedling growth attributes of maize after the control treatment compared with $8 \%$ salinity concentration. Decomposition period alone showed no differences for improving emergence parameters while 2 weeks' decomposition period improved root length, plant fresh and dry weight compared with 4 weeks.

However, the decomposition period of 2 weeks at $2 \%$ residue concentration along with $4 \%$ salinity level increased the plant biomass production by improving plant fresh weight, dry weight and root length compared with 4 weeks decomposition period at other residue concentrations and salinity levels. Higher salinity levels inhibited both germination and growth characteristics of maize.

Received 01092016

Accepted 23022017

\section{References}

1. Alam S. M., Shaikh A. H. 2007. Influence of leaf extract of nettle leaf goosefoot (Chenopodium morale L.) and $\mathrm{NaCl}$ salinity on germination and seedling growth of rice (Oryza sativa L.). Pakistan Journal of Botany, 39 (5): 1695-1699.

2. Ali I., Shah S. A., Ahmed H. M., Rehman K. U., Ahmed M. 2012. Studies on genetic diversity for seed quality in rapeseed (Brassica napus L.) germplasm of Pakistan through near infrared spectroscopy and principle component analysis. Pakistan Journal of Botany, 44: 219-222.

3. Aliu S., Rusinovci I., Fetahu S., Gashi B., Simeonovska E., Rozman L. 2015. The effect of salt stress on the germination of maize (Zea mays L.) seeds and photosynthetic pigments. Acta Agriculturae Slovenica, 105 (1): 85-94. https://doi.org/10.14720/aas.2015.105.1.09

4. Al-Sherif E., Hegazy A. K., Gomaa N. H., Hassan M. O. 2013. Allelopathic effect of black mustard tissues and root exudates on some crops and weeds. Planta Daninha, 31 (1): 11-19. https://doi.org/10.1590/S0100-83582013000100002

5. Asgharipour M. R., Armin M. 2010. Inhibitory effects of sorghum halepense root and leaf extracts on germination and early seedling growth of widely used medicinal plants. Advances in Environmental Biology, 4 (2): 316-324.

6. AOSA Rules for Testing Seeds. 1990. Association of Official Seed Analysts. 
7. Batish D. R., Lavanya K., Singh H. P., Kohli R. K. 2007. Root mediate allelopathic interference of nettle-leaved goosefoot (Chenopodium murale) on wheat (Triticum aestivum). Journal of Agronomy and Crop Science, 193: 37-44. https://doi.org/10.1111/j.1439-037X.2006.00243.x

8. Chachar Q. I., Solangi A. G., Verhoef A. 2008. Influence of sodium chloride on seed germination and seedling root growth of cotton (Gossypium hirsutum L.). Pakistan Journal of Botany, 40 (1): 183-197.

9. Chauhan J. S., Bhadauria V. P. S., Singh M., Singh K. H., Kumar A. 2007. Quality characteristics and their interrelationship in Indian rapeseed-mustard (Brassica spp.) varieties. Indian Journal of Agricultural Science, 77: 616-620. https://doi.org/10.1046/j.1439-037X.2002.00574.X

10. Coolbear P., Francis A., Grierson D. 1984. The effect of low temperature pre-sowing treatment on the germination performance and membrane integrity of artificially aged tomato seeds. Journal of Experimental Botany, 35: 1609-1617. https://doi.org/10.1093/jxb/35.11.1609

11. Devkota A., Jha P. K. 2010. Seed germination responses of the medicinal herb Centella asiatica. Brazilian Journal of Plant Physiology, 22 (2): 143-150. https://doi.org/10.1590/S1677-04202010000200008

12.Duke S. O., Wedge D. E., Cerdeira A. L., Matallo M. B 2007. Herbicide effects on plant disease. Outlooks on Pest Management, 18 (1): 36-40. https://doi.org/10.1564/18feb13

13.Ellis R. H., Roberts E. H. 1980. Towards a rational basis for testing seed quality. Hebblethwaite P. D. (ed.). Seed production. London, UK, p. 605-635.

14.Farooq M., Jabran K., Rehman H., Hussain M. 2008. Allelopathic effects of rice on seedling development in wheat, oat, barley and berseem. Allelopathy Journal, 22: $385-390$.

15.Haddadchi G. R., Gerivani Z. 2009. Effects of phenolic extracts of canola (Brassica napus L.) on germination and physiological responses of soybean (Glycin max L.) seedlings. International Journal of Plant Production, 3 (1): 63-74.

16.He H. B., Wang H. B., Fang C. X., Lin Z. H., Yu Z. M., Lin W. X. 2012. Separation of allelopathy from resource competition using rice/barnyardgrass mixed-cultures. PLOS ONE, 7 (5): e37201. https://doi.org/10.1371/journal.pone.0037201

17.Iqbal M. A., Iqbal A., Akbar N., Abbas R. N., Khan H. Z., Maqsood Q. 2014. Response of canola to foliar application of moringa (Moringaolifera L.) and brassica (Brassica napus L.) water extracts. International Journal of Agriculture and Crop Sciences, 7 (14): 1431-1433.

18. Jabeen N., Ahmed M. 2009. Possible allelopathic effects of three different weeds on germination and growth of maize (Zea mays) cultivars. Pakistan Journal of Botany, 41 (4): 1677-1683.

19. Jafariehyazdi E., Javidfar F. 2011. Comparison of allelopathic effects of some brassica species in two growth stages on germination and growth of sunflower. Plant, Soil and Environment, 57 (2): 52-56.

20.Jamil M., Lee D. B., Jung K. Y., Ashraf M., Lee S. C., Rha E. S. 2006. Effect of salt $(\mathrm{NaCl})$ stress on germination and early seedling growth of four vegetables species. Journal of Central European Agriculture, 7: 273-282.

21.Khodarahmpour Z., Ifar M., Motamidi M. 2012. Effect of $\mathrm{NaCl}$ salinity on maize (Zea mays L.) at germination and early seedling age. African Journal of Biotechnology, 11 (2): 298-304. https://doi.org/10.5897/AJB11.2624
22. Messiha N. K., Ahmed S. A., Kowthar G., Dawood M. G., El-Masry R. R. 2013. The physiological influence of allelochemicals in two Brassicaceae plant seeds on the growth and propagative capacity of Cyperus rotundus and Zea mays L. World Applied Sciences Journal, 26 (9): 1142-1149.

23. Nazir T., Uniyal A. K., Todaria N. P. 2007. Allelopathic behavior of three medicinal plant species on traditional agriculture crops of Garhwal Himalaya, India. Agroforestry Systems, 69 (3): 183-187.

https://doi.org/10.1007/s10457-006-9023-8

24. Ouda S. A. E., Mohamed S. G., Khalil F. A. 2008. Modeling the effect of different stress conditions on maize productivity using yield-stress model. International Journal of Engineering Science, 2 (1): 57-62.

25. Rahimi A. 2013. Seed priming improves the germination performance of cumin (Cuminum syminum L.) under temperature and water stress. Industrial Crops and Products, 42: 454-460.

https://doi.org/10.1016/j.indcrop.2012.06.026

26. Ranal M. A., Santana D. G., Ferreira W. R., Rodrigues C. M. 2009. Calculating germination measurements and organizing spreadsheets. Brazilian Journal of Botany, 32 (4): 849-855. https://doi.org/10.1590/S0100-84042009000400022

27. Rigon J. P. G., Capuani S., Cherubin M. R., Wastowski A. D., Rosa G. M. 2012. Allelopathic effects of aqueous extract of Brassica napus on germination of seeds of Phaseolus vulgaris. Brazilian Journal of Agricultural Sciences, 7 (3): 451-455.

https://doi.org/10.5039/agraria.v7i3a1732

28. Singh B., Uniyal A. K., Todaria N. P. 2007. Studies on allelopathic influence of Zanthoxylum armatum D.C. on important field crops seeking its sustainable domestication in existing agroforestry systems of Garhwal Himalaya, India. Journal of Sustainable Agriculture, 30 (3): 87-95. https://doi.org/10.1300/J064v30n03_07

29. Somani L. L. 2007. Plant growth in saline environment. Crop production with saline water. Jodhpur, India, p. 98-158

30. Steel R. G. D., Torrie J. H., Dickey D. A. 1997. Principles and procedures of statistics. A biometrical approach ( $3^{\text {rd }}$ ed.). New York, USA. https://doi.org/10.1002/bimj.19620040313

31.Ullah F., Sultan A. U., Wazir M., Shinwari Z. K. 2014. Phytotoxic effects of safflower yellow exposure on seed germination and early seedling growth of canola (Brassica napus L.). Pakistan Journal of Botany, 46 (5): 1741-1746.

32. Vaughn S. F., Palmquist D. E., Duval S. L. 2006. Herbicidal activity of glucosinolate-containing seed meals. Weed Science, 54: 743-748. https://doi.org/10.1614/WS-06-007R.1

33. Walsh D., Sanderson D., Hall L. M., Mugo S., Hills M. J. 2014. Allelopathic effects of camelina (Camelina sativa) and canola (Brassica napus) on wild oat, flax and radish. Allelopathy Journal, 33 (1): 83-96.

34.Zaji B., Majd A. 2011. Allelopathic potential of canola (Brassica napus L.) residues on weed suppression and yield response of maize (Zea mays L.). International Conference on Chemical, Ecology and Environmental Sciences IICCEES. Pattaya, Thailand, p. 457-460.

35.Zehtab-Salmasi S. 2008. Effects of salinity and temperature on germination of dill (Anethum graveolens L.). Plant Sciences Research, 1 (1): 27-29. 
ISSN 1392-3196 / e-ISSN 2335-8947

Zemdirbyste-Agriculture, vol. 104, No. 3 (2017), p. 259-266

DOI 10.13080/z-a.2017.104.033

\title{
Rapsų alelopatinis poveikis kukurūzų sėklų sudygimui ir daigų augimui skirtingo druskingumo substratuose
}

\author{
M. Naeem ${ }^{1}$, U. Nisar ${ }^{1}$, F. Khalid ${ }^{1}$, A. Mehmood ${ }^{1}$, H. H. Ali ${ }^{2}$ \\ ${ }^{1}$ Bahawalpur Islamia universiteto Žemės ūkio ir aplinkos mokslų universiteto koledžas, Pakistanas \\ ${ }^{2}$ Sargodha universiteto Žemès ūkio universiteto koledžas, Pakistanas
}

\section{Santrauka}

Brassica rūšys pasižymi alelopatiniu poveikiu, kuris mažina sėklų sudygimą po jų auginamų javų sẻjomainoje. Siekiant nustatyti rapsų alelopatini potencialą kukurūzų sẻklų sudygimui ir daigų augimui esant skirtingiems druskingumo lygiams, eksperimentai atlikti laboratorijoje ir šiltnamyje. Laboratorinį eksperimentą sudarè du veiksniai: augalo dalių - šaknų, daigų, lapų - bei viso augalo ekstraktai ir įvairios jų koncentracijos $(0,5$, 10, 15 ir $20 \%$ ). Eksperimente šiltnamyje tirti trys veiksniai: skirtingi druskingumo lygiai $(0,4$ ir $8 \%$ ), augalų liekanų koncentracijos (0,2 ir $4 \%$ ) ir irimo laikas (2 ir 4 savaitės). Eksperimento variantai išdèstyti taikant visišką rendomizaciją, su penkiais pakartojimais daigumo eksperimente Petri lèkštelèse ir trimis pakartojimais vegetaciniame eksperimente, augalus auginant induose su skirtingu kiekiu rapsų liekanų dirvožemyje.

Tyrimo rezultatai parodé, kad kukurūzų sẻklų sudygimui augalo ịvairių dalių ekstraktai neturèjo ịtakos, o skirtingos jų koncentracijos turejo esminès ịtakos. Augimą reikšmingai veikè įvairių augalo dalių ekstraktų ir ịvairių koncentracijų sąveika. Šaknų ir lapų $5 \%$ koncentracijos ekstraktai lèmè maksimalų šaknų ilgị, daigų ilgị ir sausųjų medžiagų kiekį, palyginus su kitų augalo dalių ekstraktais arba kitomis koncentracijomis. Antrojo šiltnamyje atlikto eksperimento rezultatai parodé, kad kukurūzų sẻklų daigumui reikšmingos įtakos neturèjo nei augalų dalys, nei jų irimo laikas. Tačiau didinant druskos tirpalo koncentraciją druskingumo lygiai slopino kukurūzų sėklų dygimą. Didžiausias žalios masės ir sausujų medžiagų kiekis buvo gautas rapsų liekanas paveikus $2 \%$ koncentracijos ekstraktu kartu su 4 \% druskos koncentracijos tirpalu. Rapsai turejo alelopatinị poveikị kukurūzų sẻklų sudygimui ir daigų augimui, tačiau didinant druskingumą ši įtaka palaipsniui mažejo.

Reikšminiai žodžiai: alelopatija, augimo skatinimas, Brassica napus, Zea mays. 